\title{
Globin Domain Interactions Control Heme Pocket Conformation and Oligomerization of Globin Coupled Sensors
}

Shannon Rivera, Justin L. Burns, Gregory E. Vansuch, Bryant Chica, Emily E. Weinert*

*Corresponding author: emily.weinert@emory.edu; 404-712-6865; Department of Chemistry, Emory University, 1515 Dickey Drive, Atlanta, GA 30322 


\section{$\underline{\text { Abstract }}$}

Globin coupled sensors (GCS) are $\mathrm{O}_{2}$-sensing proteins used by bacteria to monitor the surrounding gaseous environment. To investigate the biphasic $\mathrm{O}_{2}$ dissociation kinetics observed for full-length GCS proteins, isolated globin domains from Pectobacterium carotovorum ssp. carotovorum (PccGlobin), and Bordetella pertussis (BpeGlobin), have been characterized. PccGlobin is found to be dimeric, while BpeGlobin is monomeric, indicating key differences in the globin domain dimer interface. Through characterization of wild type globin domains and globin variants with mutations at the dimer interface and within the distal pocket, dimerization of the globin domain is demonstrated to correlate with biphasic dissociation kinetics. Furthermore, a distal pocket tyrosine is identified as the primary hydrogen bond donor, while a secondary hydrogen bond donor within the distal heme pocket is involved in conformation(s) that lead to the second $\mathrm{O}_{2}$ dissociation rate. These findings highlight the role of the globin dimer interface in controlling properties of both the heme pocket and full-length GCS proteins.

Keywords: Globin coupled sensor; oxygen sensor; globin domain; oligomerization state

\section{Introduction}

Bacteria have evolved sophisticated systems to sense changes in their environment and to respond, optimizing their survival ${ }^{1-2}$. The mechanism by which bacteria sense changing conditions and transmit those signals into metabolic and phenotypic responses is an active area

of research. The ability of bacteria to adjust to changing $\mathrm{O}_{2}$ concentrations ${ }^{3-8}$ suggests signaling pathway(s) that can sense extracellular $\mathrm{O}_{2}$ and adjust intracellular chemistry. A class of heme 
proteins, termed globin coupled sensors, is predicted to serve as bacterial environmental $\mathrm{O}_{2}$ sensors within a large number of bacteria ${ }^{5,9-11}$. Globin coupled sensors (GCS) consist of a sensor globin domain linked to an output domain by a variable middle domain. Output domains vary widely and include methyl accepting chemotaxis proteins (MCP) ${ }^{12-14}$, histidine kinases ${ }^{15}$, antianti-sigma factors ${ }^{16}$, and diguanylate cyclases ${ }^{5,17-20}$, with methyl accepting chemotaxis proteins and diguanylate cyclases being the most prevalent.

Within the cell, diguanylate cyclases are found fused to many types of regulatory domains and are responsible for cyclizing two GTP to form c-di-GMP, a bacterial secondary messenger that controls biofilm formation ${ }^{21-22}$. Diguanylate cyclases have been demonstrated to be catalytically active as dimers, with the cyclization reaction occurring across the dimer interface ${ }^{23-27}$, regardless of their regulatory domain. Work on the diguanylate cyclase-containing GCS proteins from E. coli $(E c \text { DosC })^{18,20,28}$, Desulfotalea psychrophila $(\mathrm{HemDGC})^{19}$, Bordetella pertussis (BpeGReg, the causative agent of whooping cough) ${ }^{17,29}$, and Pectobacerium carotovorum ssp. carotovorum (PccGCS, a plant pathogen) ${ }^{17}$ has demonstrated that cyclase activity is increased upon binding of $\mathrm{O}_{2}$ to the globin domain, as compared with the $\mathrm{Fe}^{\mathrm{II}}$ unligated state. Further characterization of GCS proteins from $B$. pertussis and $P$. carotovorum found that full length GCS proteins form mixtures of oligomeric states (monomer-dimer-tetramer and dimer-tetrameroctamer, respectively) ${ }^{17}$. For both $P c c$ GCS and BpeGReg, the tetrameric assemblies were found to exhibit the highest cyclase activity (on a per monomer basis), individual oligomeric assemblies were slow to re-equilibrate (>18 hrs.), and oligomer percentages were not dependent on protein or salt concentration, suggesting that PccGCS and BpeGReg exist as mixtures of kinetically trapped oligomers ${ }^{17}$. Furthermore, a shift toward tetrameric assemblies, which exhibit 
increased catalytic activity, was triggered by $\mathrm{O}_{2}$ binding to the heme ${ }^{17}$, highlighting the role of the sensor globin domain in controlling protein conformation/oligomerization. Characterization of $E c$ DosC ${ }^{28}$ and HemDGC ${ }^{19}$ also showed that the proteins formed primarily dimeric and tetrameric assemblies, respectively, highlighting that GCS oligomerization may be conserved.

Structures of isolated sensor globin domains from Bacillus subtilis (HemAT-Bs, MCP output domain $)^{30}$ and $E c$ DosC ${ }^{28} \mathrm{GCS}$ proteins have been solved and shown to form dimers in the crystals (homology model of PccGlobin based on HemAT-Bs is shown in Fig. 1). The globin domains maintain a primarily $\alpha$-helical globin fold with dimer interfaces that consist of two helices from each monomer, arranged in coiled-coil packing with key hydrophobic and electrostatic interactions helping to stabilize the dimer ${ }^{28,30}$. Within the heme pocket, a conserved proximal histidine ligates to the heme, and a distal tyrosine helps to stabilize bound ligands (Fig. 1A). The HemAT-Bs globin distal pocket has a second distal pocket hydrogen bond donor, a threonine, that also interacts with bound ligands ${ }^{30-32}$, while the $E c$ DosC globin only has a distal pocket tyrosine ${ }^{18,28}$. In contrast, the prototypical histidyl-ligated heme protein, myoglobin, contains a distal pocket histidine that coordinates bound ligands. Changes in oxidation/ligation states resulted in subtle rearrangements within the sensor globin structures, such as slight rotation at the dimer interface, which are hypothesized to lead to the observed ligand-dependent changes in catalytic activity ${ }^{30}$. However, to date, the effects of dimerization on GCS globin domain structure and conformation are still unknown. 

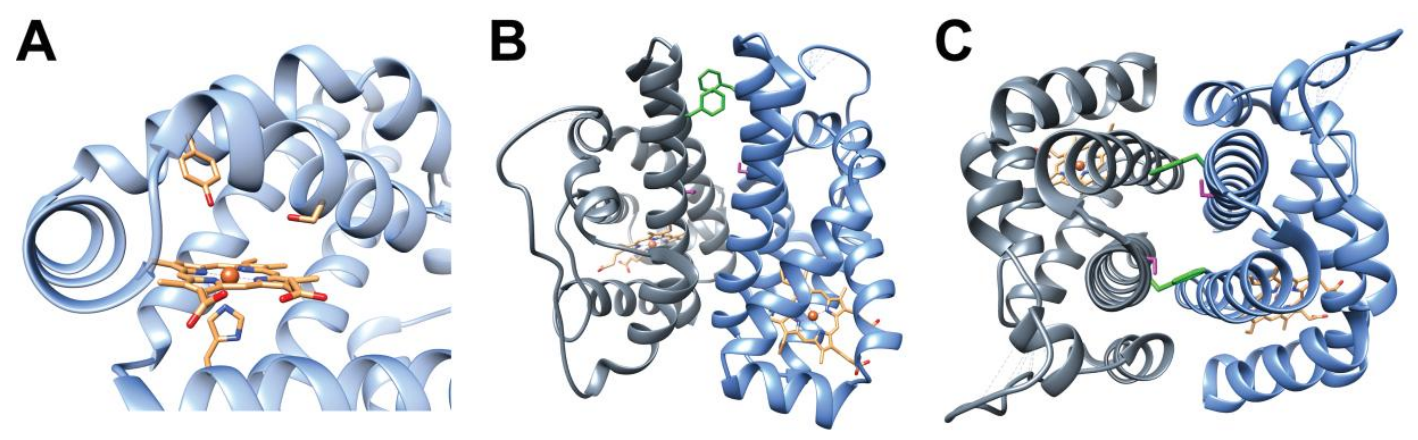

Figure 1. Homology model of PccGlobin (based on PDB ID: $10 R 4{ }^{30}$ ). A) Heme pocket with proximal histidine and distal tyrosine and serine highlighted. B) Dimer interface with F148R shown in green and S155R shown in pink. C) Alternate view of the putative dimer interface.

Previous spectroscopic characterization of HemAT-Bs observed biphasic $\mathrm{O}_{2}$ dissociation kinetics ${ }^{32}$, which, through resonance Raman studies ${ }^{31}$, were attributed to multiple open and closed conformations of the heme pocket. In addition, $\mathrm{O}_{2}$ dissociation from dimeric and tetrameric assemblies of $P c c \mathrm{GCS}$ and BpeGReg was found to be biphasic ${ }^{17}$, again suggesting multiple conformations, and possibly hydrogen bonding patterns, within GCS heme pockets. To investigate the role of sensor globin domains in dimerization of full-length GCS proteins and the effects of globin-globin interactions on $\mathrm{O}_{2}$ binding kinetics and hydrogen bonding interactions, isolated globin domains from PccGCS and BpeGReg were investigated to determine if globin domains are important determinants of full-length GCS oligomerization and the effects of oligomerization on the heme pocket.

\section{Materials \& Methods}

2.1. Materials. All chemicals, PCR-related enzymes, and desalting columns used in these experiments were purchased from Sigma-Aldrich, RPI, Bio-Life, GE Healthcare, and Bio-Rad. 
Analytical gel filtration columns were manufactured by Agilent (SEC $3 \mu \mathrm{m}, 150 \AA$, $4.6 x 300 \mathrm{~mm}$ and SEC $3 \mu \mathrm{m}, 300 \AA ̊$, 7.8x300 mm HPLC columns).

2.2. Mutagenesis. Codon-optimized genes for BpeGReg and PccGCS in pET-20b ${ }^{17}$ served as templates for site-directed mutagenesis (mutagenic primers are listed in Table S1). Positive transformants of all constructs were screened on LB plates containing $100 \mu \mathrm{g} / \mathrm{mL}$ ampicillin and the DNA sequences were confirmed by sequencing. Globin constructs were generated based on the length of the globin domain predicted by BLAST ${ }^{33}$ and based on homology models. Stop codons were introduced at position 176 of PccGCS and position 161 of BpeGReg. A BpeGlobin construct truncated at position 156 also was generated and analyzed; however, this construct was unstable, suggesting that the globin domain extends further than predicted by BLAST. PCR mutagenesis conditions were as follows: $95^{\circ} \mathrm{C}$ for $1: 00 \mathrm{~min}$; $\left(95^{\circ} \mathrm{C}\right.$ for $0: 50 \mathrm{~min}, 55^{\circ} \mathrm{C}$ for $0: 40$ $\min , 68^{\circ} \mathrm{C}$ for $6: 00 \mathrm{~min}$ ), repeated 16 times; $68^{\circ} \mathrm{C}$ for 7:00 min.

2.3. Expression and Purification. Proteins were expressed and purified as previously described ${ }^{17}$, with minor modifications. Globin domain expressions were induced with $100 \mu \mathrm{M}$ IPTG for $\sim 20$ hours at $25{ }^{\circ} \mathrm{C}$ before being harvested via centrifugation. Cell pellets were resuspended in Buffer A (50 mM Tris, $300 \mathrm{mM} \mathrm{NaCl,} \mathrm{5 \%} \mathrm{Glycerol} \mathrm{(v/v),} 20 \mathrm{mM}$ imidazole, $\mathrm{pH}$ 7.0), lysed using a homogenizer (Avestin, Inc.) and the resulting lysates were centrifuged at 130,000 $\mathrm{x} g$ in a Beckman Optima L-90X ultracentrifuge at $4{ }^{\circ} \mathrm{C}$ for 1 hour. Supernatants were applied to a preequilibrated HisPur Ni-column (Fisher Scientific) and proteins were eluted with Buffer B (buffer A with $250 \mathrm{mM}$ imidazole, $\mathrm{pH}$ 7.0). Purified proteins (with intact hexa-histidine tag) were desalted using a S200 gel filtration column (GE Healthcare) into Buffer C (50 mM Tris, $50 \mathrm{mM}$ $\mathrm{NaCl}, 1 \mathrm{mM}$ DTT, 5\% Glycerol (v/v), $\mathrm{pH}$ 7.0). Proteins were collected and concentrated via ultrafiltration (YM-10, $10 \mathrm{kDa}$ MWCO filter, Millipore), aliquoted, flash frozen, and stored at - 
$80{ }^{\circ} \mathrm{C}$ until use. Protein identity and purity were verified by SDS-PAGE (Fig. S1) and MALDI mass spectrometry (Table S2).

2.4. Electronic Absorption Spectroscopy. All spectra were recorded on an Agilent Cary 100 with Peltier accessory. Preparation of complexes was carried out as previously described except that the proteins were prepared in Buffer D (50 mM Tris, $50 \mathrm{mM} \mathrm{NaCl}, 1 \mathrm{mM}$ DTT, pH 7.0) ${ }^{17,34-35}$.

2.5. Analytical Gel Filtration. Samples were analyzed as previously described ${ }^{17}$. Briefly, globin constructs were detected via size exclusion chromatography using an Agilent 1200 infinity system with an Agilent SEC-300 (7.8 mm X 300 mm, $300 \AA$ and $4.6 \mathrm{~mm}$ X $300 \mathrm{~mm}, 150 \AA)$ and diode array detector (simultaneous detection at 214, 416, and $431 \mathrm{~nm}$ ). Proteins were reduced in an anaerobic chamber and then allowed to bind $\mathrm{O}_{2}$ following mixing with aerobic buffer before injection onto SEC-300 column. The mobile phase for all experiments consisted of $150 \mathrm{mM}$ sodium phosphate, $\mathrm{pH} 7.0$, with varying concentrations of $\mathrm{NaCl}(0-300 \mathrm{mM})$. Spectra were collected for each peak during the SEC run to confirm that the heme remained in the appropriate ligation state. Globular proteins (Sigma-Aldrich) consisting of thyroglobulin (669 kDa), ferritin (443 kDa), $\beta$-amylase (200 kDa), alcohol dehydrogenase (150 kDa), bovine serum albumin (66 $\mathrm{kDa})$, carbonic anhydrase $(29 \mathrm{kDa})$ and myoglobin $(16.7 \mathrm{kDa})$ were used as molecular weight standards for calibration curves. PccGlobin and BpeGlobin were analyzed at various concentrations $(5,10,50,100,200 \mu \mathrm{M})$ while the globin mutants were analyzed at $100 \mu \mathrm{M}$.

2.6. Stopped Flow UV-Visible Spectroscopy. $\mathrm{O}_{2}$ dissociation rates were performed as previously described ${ }^{17,35}$. Globins were reduced with dithionite in an anaerobic chamber (Coy), desalted into Buffer D, and then mixed with $\mathrm{O}_{2}$ saturated buffer to yield final concentrations of 5-10 $\mu \mathrm{M}$. Protein samples were rapidly mixed with a solution of sodium dithionite in Buffer D (final 
dithionite concentration $=5 \mathrm{mM}$; dithionite concentration of $0.5 \mathrm{mM}$ also was tested and dithionite concentration was found to not affect the $\mathrm{O}_{2}$ dissociation rate) in an SX20 stopped flow apparatus. The dissociation of $\mathrm{O}_{2}$ from globin constructs at $25^{\circ} \mathrm{C}$ was monitored using the SX20 stopped flow equipped with a diode array detector and temperature controlled bath, and fit globally using Pro-KII software (Applied Photophysics). Additional fitting analysis was performed using Igor Pro (Wavemetrics).

2.7. Laser Flash Photolysis. Oxygen association rates were measured as previously described ${ }^{16,}$ 35-36. $\mathrm{Fe}^{\mathrm{II}}-\mathrm{O}_{2}$ complexes of globin constructs were generated in Buffer D as described above (Section 2.4). Transient visible spectra were collected at room temperature on a home-built nanosecond transient infrared system described elsewhere with minor modifications ${ }^{35-36}$. Briefly, a Surelight $^{\mathrm{TM}} \mathrm{Nd}$ :YAG (Continuum, Inc.) $1064 \mathrm{~nm}$ fundamental was frequency doubled and the resulting $532 \mathrm{~nm}$ pump power adjusted to $65 \mu \mathrm{J} /$ pulse and the light focused to a $\sim 1.3 \mathrm{~mm}$ diameter spot. The $405 \mathrm{~nm}$ probe (Thor Labs, Inc.) was focused onto a silicon avalanche photodetector (Thor Labs, Inc.). To calculate $\Delta \mathrm{A}$, the pre-photolysis signal was averaged and used as $\mathrm{I} 0$, and the pumped signal was used as $\mathrm{I}(\Delta \mathrm{A}=-\log (\mathrm{I} / \mathrm{I} 0))$. Transient visible spectra were analyzed with IGOR Pro 5.00 (Wavemetrics, Inc.). Reported rates are the average of three rates from triplicate measurements and the error reported as +/- the standard deviation of the three rates.

2.8. Protein Crosslinking. Proteins were incubated with $\mathrm{BS}^{3}$ crosslinker (Thermo Scientific) for 45 minutes at room temperature in $1: 1,1: 10,1: 20,1: 50,1: 100$ and 1:200 (protein:crosslinker) concentration ratios. Reactions were quenched by adding $0.5 \mathrm{M}$ ammonium bicarbonate and letting the reaction incubate for an additional 20 minutes. The protein samples were then mixed 
with 6X SDS loading buffer and heat denatured at $90{ }^{\circ} \mathrm{C}$ for 15 minutes before being run on a Criterion TGX 4-20\% gradient gel (Bio-Rad).

\section{Results and Discussion}

Previous studies found that GCS proteins from $P$. carotovorum and B. pertussis exist as mixtures of oligomeric states, with the dimeric and tetrameric assemblies having different catalytic activity, but consistent $\mathrm{O}_{2}$ dissociation rates, for each respective GCS ${ }^{17}$. However, the interfaces between domains and molecular effects of oligomerization are currently unknown. To identify the effects of oligomerization on the heme pocket and ligand binding, truncations were made to PccGCS and BpeGReg to yield isolated globin domains (PccGlobin and BpeGlobin, respectively). In addition, mutations of putative distal pocket residues (tyrosine and serine; BpeGlobin(S68A) and (Y43A), PccGlobin(S82A) and (Y57A)) were generated to investigate the roles of hydrogen bonding patterns in $\mathrm{O}_{2}$ dissociation kinetics (Fig. 1A). Finally, mutations were introduced at the putative globin dimer interface (PccGlobin(F148R) and (S155R)) to weaken globin-globin interactions and further investigate the role(s) of globin dimerization on ligand binding properties (Fig. 1B and 1C).

\subsection{Heme spectra of globin constructs}

PccGlobin and BpeGlobin WT and mutant constructs were expressed, purified, and characterized by UV-visible spectroscopy. Both proteins exhibit spectra that are characteristic of histidylligated heme proteins and are nearly identical to the respective full-length proteins ${ }^{17,29}$ (Fig. 2A, 2B) and to other previously characterized sensor globins 14, 19-20, 29 . Similarly, ferrous PccGlobin(S82A), BpeGlobin(S68A), and PccGlobin(F148R) were able to bind all of the 
A

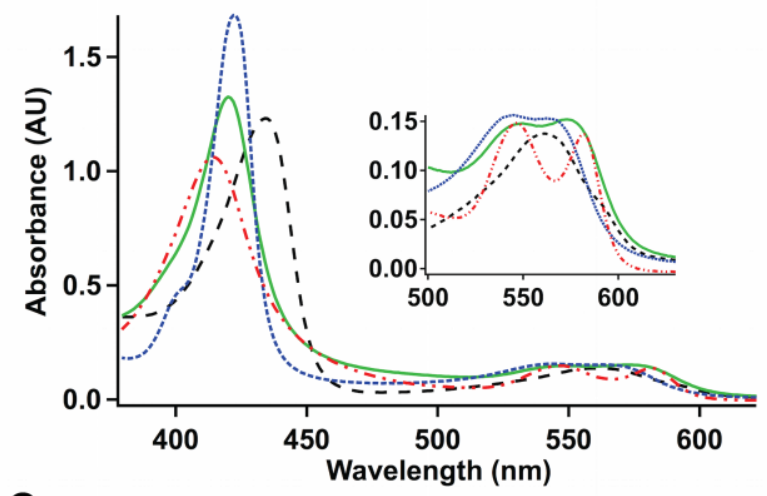

C

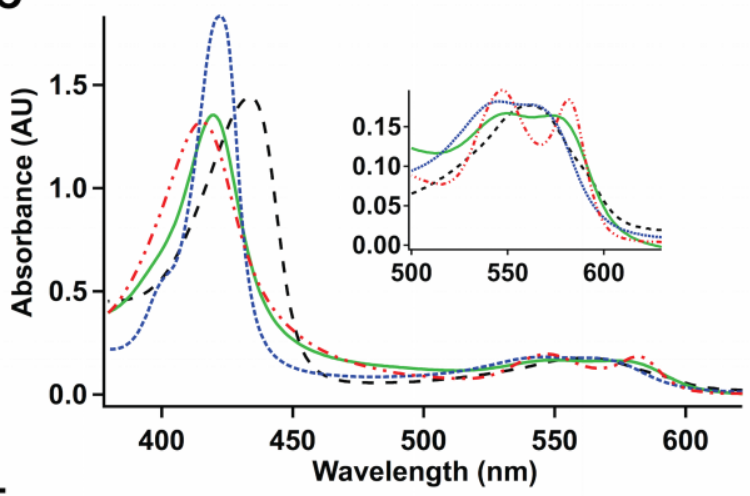

E

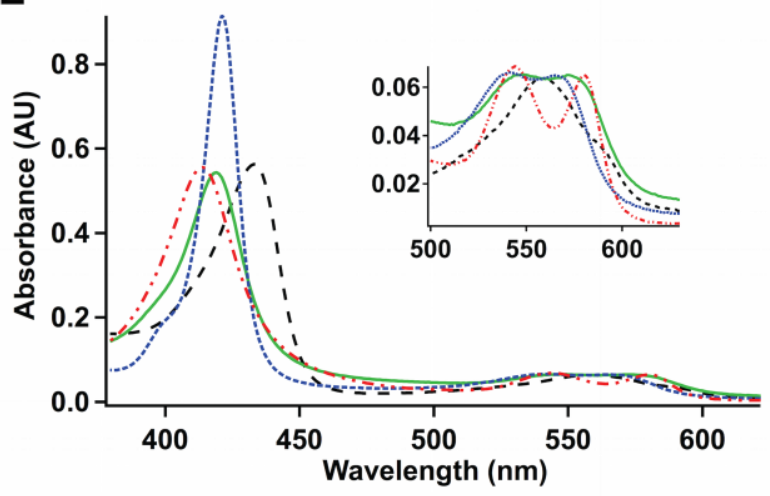

B

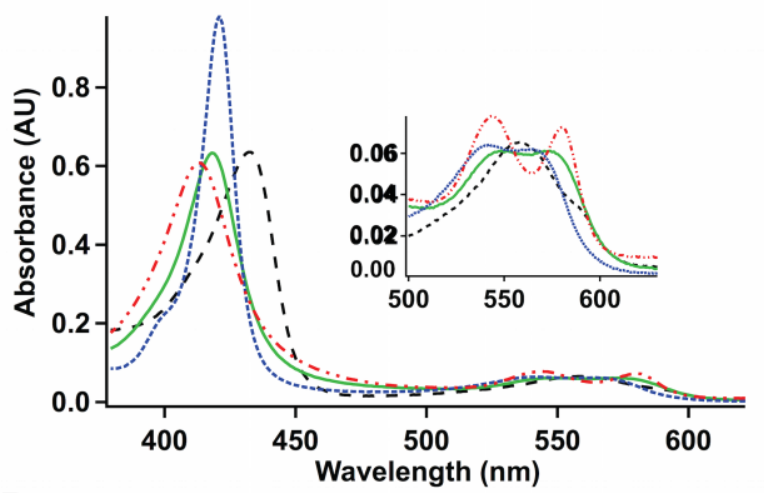

D

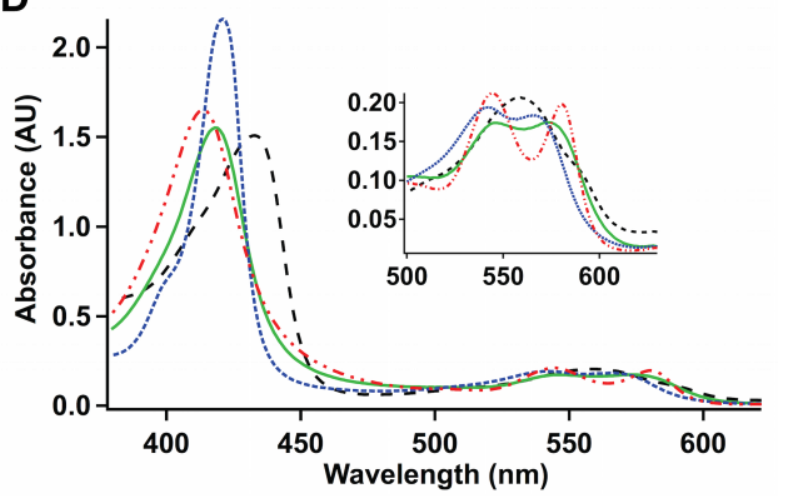

Figure 2. UV-visible spectra of $P c c$ Globin and BpeGlobin proteins $(50 \mathrm{mM}$ Tris, $50 \mathrm{mM} \mathrm{NaCl}$, 1 mM DTT, pH 7.0; 5-10 $\mu$ M protein. Black, $\mathrm{Fe}^{\mathrm{II}}$; red, $\mathrm{Fe}^{\mathrm{II}}-\mathrm{O}_{2}$; blue, $\mathrm{Fe}^{\mathrm{II}}-\mathrm{CO}$; green, $\mathrm{Fe}^{\mathrm{II}}-\mathrm{NO}$. A) PccGlobin WT. B) BpeGlobin WT. C) PccGlobin(S82A). D) BpeGlobin(S68A). E) PccGlobin(F148R). Absorbance maxima and extinction coefficients are listed in Table S3. 
diatomic ligands $\left(\mathrm{CO}, \mathrm{NO}, \mathrm{O}_{2}\right)$ and exhibited spectra nearly identical to their respective WT globin constructs (Fig. 2C-E). In contrast, both tyrosine mutants, PccGlobin(Y57F) and BpeGlobin(Y43F), rapidly oxidized when exposed to $\mathrm{O}_{2}$, with no detectable stable $\mathrm{O}_{2}$ binding, identifying the distal pocket tyrosine residues as the predominant hydrogen bond donors.

\subsection{Oligomeric states of WT globin constructs}

Due to previous studies demonstrating that GCS proteins oligomerize ${ }^{17}$, analytical gel filtration (AGF) was performed to determine the accessible oligomerization states. Both WT globin proteins were found to form predominantly (>90\%) one oligomeric state, dimer for PccGlobin (with $<5 \%$ tetramer) and monomer for BpeGlobin (with $<5 \%$ dimer) (Fig. 3A). Similar to fulllength PccGCS and BpeGReg proteins, PccGlobin and BpeGlobin (36\% identical, 61\% similar) access different oligomeric states, despite the lack of obvious differences in amino acid similarity at the putative dimer interface (Fig. S2).

The dimerization affinities of WT PccGlobin and BpeGlobin were interrogated by changing protein and salt concentrations. From 5-200 $\mu \mathrm{M}$ protein concentration, only minor shifts $(<10 \%)$ in the oligomerization state of the proteins were observed and BpeGlobin did not form significant amounts of dimeric species even at the highest protein concentrations (Fig. 3B, 3C). Furthermore, a small change was observed for PccGlobin at different salt concentrations ( $\sim 6 \mathrm{kDa}$ based on retention time), but the PccGlobin dimer interface could not be disrupted, even at 300 $\mathrm{mM} \mathrm{NaCl}$ (Fig. 4A). 


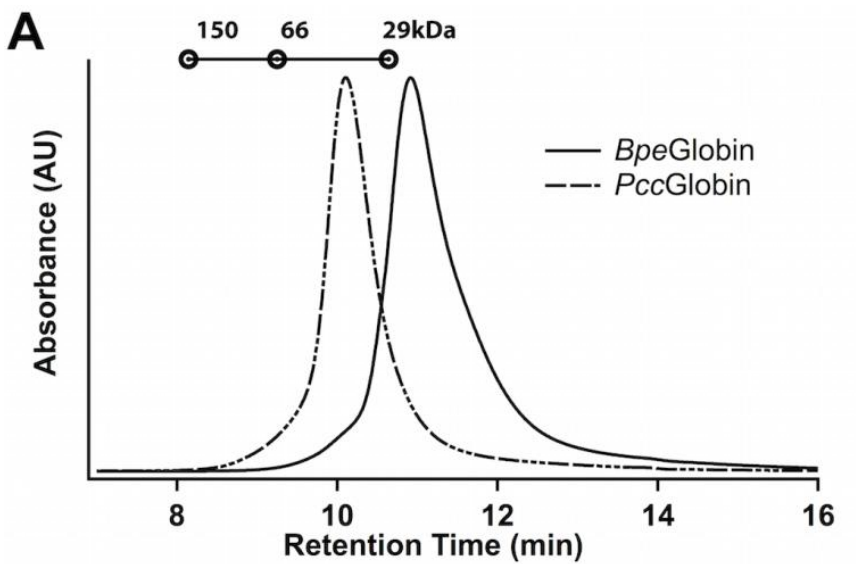

Figure 3. $P c c$ Globin and BpeGlobin oligomerization states $(50 \mathrm{mM}$ Tris, $50 \mathrm{mM}$ $\mathrm{NaCl}, 1 \mathrm{mM}$ DTT, pH 7.0). Retention times for globular standards (molecular weight listed) are plotted above each graph. A) Comparison of $\mathrm{Fe}^{\mathrm{II}}-\mathrm{O}_{2} P c c$ Globin (dashed,

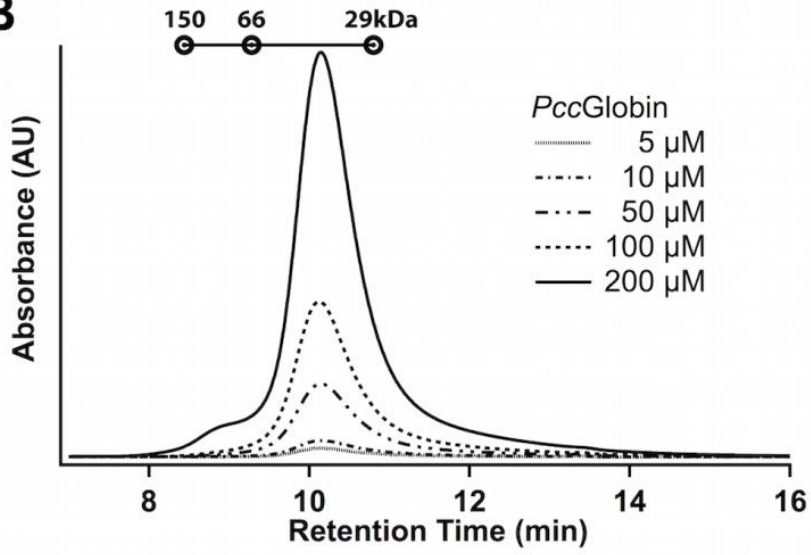
dimer) and $\mathrm{Fe}^{\mathrm{II}}-\mathrm{O}_{2} \quad$ BpeGlobin (solid, monomer). B) $\quad P c c$ Globin $\quad\left(\mathrm{Fe}^{\mathrm{II}}-\mathrm{O}_{2}\right)$ oligomerization state does not depend on concentration. C) BpeGlobin $\quad\left(\mathrm{Fe}^{\mathrm{II}}-\mathrm{O}_{2}\right)$ oligomerization state does not exhibit

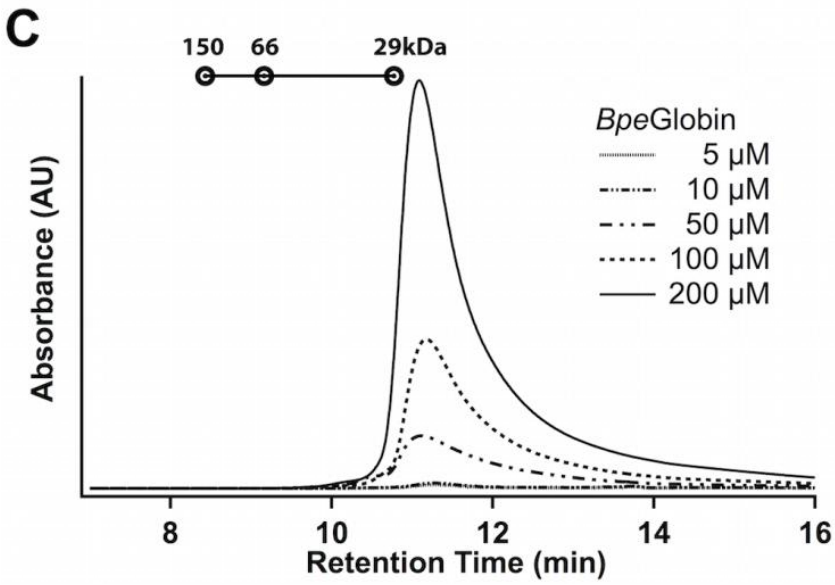
concentration dependence.

These results show that the globin domains exhibit similar properties to the full-length GCS proteins, namely that oligomerization state is not significantly modulated by protein concentration or ionic strength ${ }^{17}$. Additionally, neither PccGlobin nor BpeGlobin shifted oligomeric states when examined under anaerobic conditions; therefore $\mathrm{O}_{2}$ binding is not responsible for altering protein conformation to allow globin dimerization (Fig. 4B). Similarly, anaerobic AGF analysis of full-length PccGCS demonstrated that $\mathrm{O}_{2}$ binding controlled the dimer-tetramer-octamer/high molecular weight 
(HMW) equilibrium, but $\mathrm{Fe}^{\mathrm{II}} P c c \mathrm{GCS}$ did not yield any measureable monomer formation (J. Burns, S. Rivera, and E.E. Weinert; manuscript under review). Therefore, it is likely that $\mathrm{O}_{2}$ binding causes conformational changes that propagated through the full-length GCS proteins, altering protein-protein interactions and altering oligomer affinity without changing globin domain dimerization.
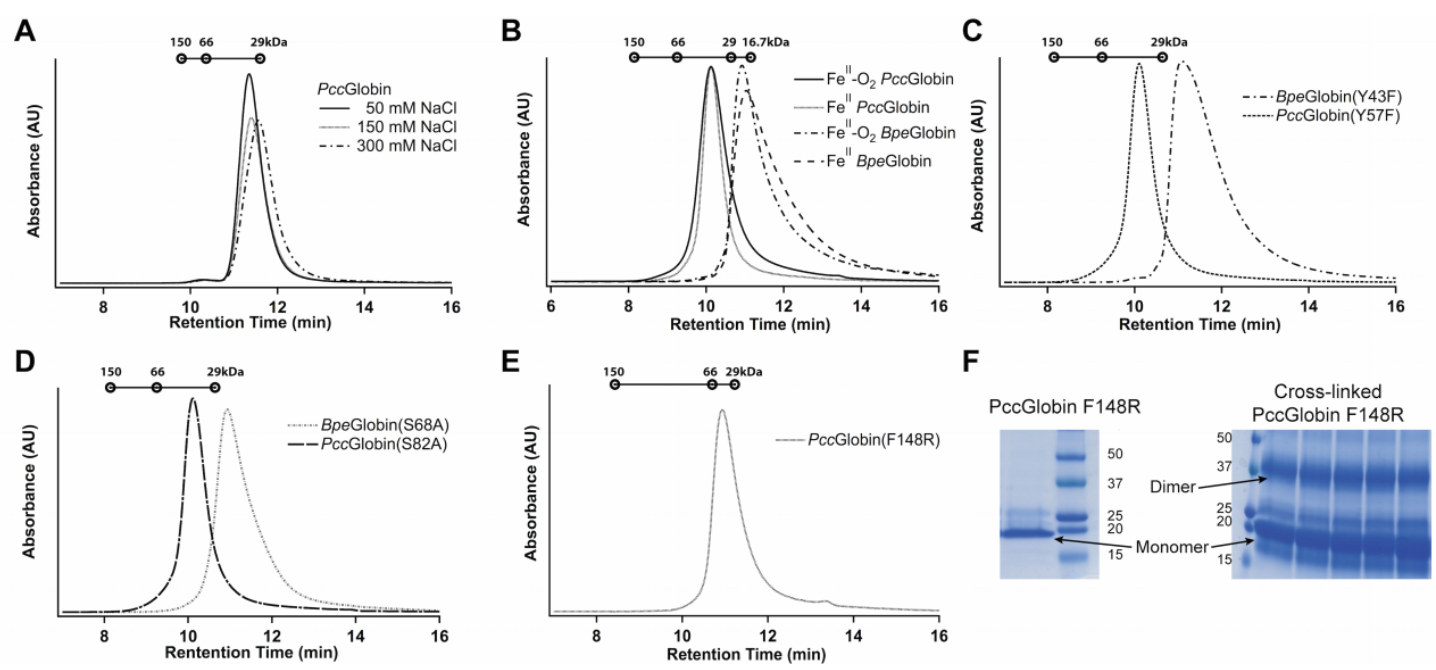

Figure 4. Salt, $\mathrm{O}_{2}$, and mutation-dependent oligomerization. Retention times for globular standards (molecular weight listed) are plotted above each graph. A) Pcc Globin $\mathrm{Fe}^{\mathrm{II}}-\mathrm{O}_{2}$ remains dimeric at a range of salt concentrations. B) PccGlobin $\mathrm{Fe}^{\mathrm{II}}$ exists as a dimer and BpeGlobin Fe ${ }^{\mathrm{II}}$ exists as a monomer. C) Mutation of the distal pocket tyrosine does not affect oligomerization state (PccGlobin and BpeGlobin $\mathrm{Fe}^{\mathrm{III}}$, due to rapid oxidation in air). D) $\mathrm{Fe}^{\mathrm{II}}-\mathrm{O}_{2} P c c$ Globin and BpeGlobin distal pocket serine mutants maintain WT oligomerization states. E) $P c c$ Globin(F148R) $\left(\mathrm{Fe}^{\mathrm{II}}-\mathrm{O}_{2}\right)$ mutation weakens dimer affinity. F) Non-crosslinked PccGlobin runs as a monomer by denaturing PAGE (left), while addition of $\mathrm{BS}^{3}$ crosslinking reagent results in formation of a clear dimer band (right). 
These data suggest that the globin domains are key determinants of the ability of full-length GCS proteins to exist as monomeric proteins. BpeGlobin is monomeric and BpeGReg is observed in the monomeric state and exists as a monomer-dimer-tetramer mixture ${ }^{17}$. In contrast, Pcc Globin is dimeric, and PccGCS has not been observed as a monomer; instead, PccGCS exists as a dimer-tetramer-octamer/HMW mixture ${ }^{17}$. Strong interactions between globin domains in PccGCS likely preclude formation of monomeric PccGCS, while weak interactions in BpeGlobin readily allow BpeGReg monomer formation. As less than 5\% of PccGlobin forms tetrameric assemblies, additional interactions clearly are required to generate higher order oligomers (tetramers and octaomers); however, globin domain dimer affinity controls the accessibility of monomeric full-length proteins.

\subsection{Characterization of globin mutants}

As the oligomerization states of $P c c$ Globin and BpeGlobin could not be altered by protein or salt concentration, weakening the globin-globin interface was probed through mutagenesis. Using a homology model based on crystal structures of other sensor globins ${ }^{28,30,37}$, residues putatively lining the interface were identified (Fig. 1B, 1C, S2). Phenylalanine148 in PccGlobin was chosen for mutagenesis as it is proposed to face directly into the coiled-coil of the dimer interface. Introduction of the F148R mutation in PccGlobin resulted in a shift to monomeric protein, as assessed by AGF (Fig. 4E). However, further analysis using protein-protein crosslinking found that PccGlobin F148R still was able to form a dimer in solution (>35\% dimer, based on cross-linking gel integration), although with significantly reduced dimer affinity (Fig. 4F). 
PccGlobin(S82A) and BpeGlobin(S68A) remained dimeric and monomeric, respectively, with no change as compared to their corresponding WT globin (Fig. 4D). In addition, from the AGF traces, both Tyr mutations remained in the same oligomerization state (dimer and monomer, respectively) as the respective WT globin (Fig. 4C). Taken together, these studies demonstrate that mutations within the heme pocket that alter hydrogen-bonding patterns do not affect oligomerization state of the globin domains. In addition, PccGlobin dimer affinity can be modulated through introduction of charged residues at the putative dimer interface, which supports the homology model structure and further highlights the high affinity of PccGlobin dimers.

\subsection{Effects of Dimerization on Oxygen Binding Kinetics}

Previous work on full-length PccGCS and BpeGReg found oligomerization-dependent effects on both cyclase activity and the two $\mathrm{O}_{2}$ dissociation rates (Table 1$)^{17}$. The different oligomerization states of the isolated globin domains provided a model system to interrogate the role of the globin-globin interface in altering $\mathrm{O}_{2}$ binding kinetics. Comparing $k_{\text {off }}$ rates $\left(k_{1}\right.$ and $\left.k_{2}\right)$ previously obtained for PccGCS and BpeGReg ${ }^{17}$ to PccGlobin and BpeGlobin, respectively, it can be noted that dissociation rates do not significantly change from full-length protein to globin truncation (Table 1, Fig. S3). However, BpeGlobin exhibits a loss of the fast rate $\left(k_{2}\right)$, while PccGlobin exhibits a large decrease in fast rate percentage (44\% vs. $21 \%$ for PccGCS and PccGlobin, respectively). The complete loss of $k_{2}$ for BpeGlobin, compared to the biphasic $\mathrm{O}_{2}$ dissociation kinetics of PccGlobin, suggests that the globin domain dimer interface is required for effective formation of the conformation(s) that lead to the fast rate. In addition, the decrease in the amplitude of $k_{2}$ observed for $P c c$ Globin demonstrates that the remaining two domains in the full- 
length GCS proteins play important roles in altering heme pocket conformation by contributing to a state with decreased $\mathrm{O}_{2}$ stabilization, thereby contributing to the fast rate. Removal of the middle and output domains of HemAT- $B s$ also resulted in changes in $\mathrm{O}_{2}$ dissociation and loss of the heme pocket closed conformation ${ }^{31-32}$, supporting roles for these domains in affecting the heme pocket.

Table 1. $\mathrm{O}_{2}$ binding kinetics $\left(k_{\mathrm{on}}, \mathrm{O}_{2}\right.$ association rate; $k_{1}$ and $k_{2}, \mathrm{O}_{2}$ dissociation rates; $\% k_{1}$ and $\% k_{2}$, amplitude of each respective $\mathrm{O}_{2}$ dissociation rate). Significant oligomeric states $(\geq 10 \%)$ in the solution of each protein during binding measurements are listed.

\begin{tabular}{|c|c|c|c|c|c|c|c|c|}
\hline Protein & $\begin{array}{c}\text { Oligomeric } \\
\text { state(s) }\end{array}$ & $\begin{array}{c}k_{\text {on }} \\
\left(\mu \mathbf{M}^{-1} \mathrm{~s}^{-1}\right)\end{array}$ & $\begin{array}{c}k_{1} \\
\left(\mathrm{~s}^{-1}\right)\end{array}$ & $\begin{array}{c}k_{2} \\
\left(\mathrm{~s}^{-1}\right)\end{array}$ & $\% k_{1}^{a}$ & $\% k_{2}^{a}$ & \multicolumn{2}{|c|}{$\begin{array}{c}K_{\mathbf{D}} \\
(\mathbf{n M})\end{array}$} \\
\hline BpeGReg & $\begin{array}{l}\text { Monomer, } \\
\text { dimer, tetramer }\end{array}$ & $7.0^{b}$ & $0.82 \pm 0.01^{\mathrm{c}}$ & $6.3 \pm 0.1^{c}$ & $39^{c}$ & $60^{c}$ & 117 & 900 \\
\hline BpeGlobin & Monomer & $17.6 \pm 0.8$ & $1.18 \pm 0.04$ & - & 100 & - & 67 & - \\
\hline $\begin{array}{l}\text { BpeGlobin } \\
\text { (S68A) }\end{array}$ & Monomer & $20.3 \pm 0.5$ & $2.08 \pm 0.01$ & - & 100 & - & 102 & - \\
\hline $\begin{array}{l}\text { BpeGlobin } \\
\text { (Y43F) }\end{array}$ & Monomer & - & - & - & - & - & - & - \\
\hline PccGCS & $\begin{array}{l}\text { Dimer, } \\
\text { tetramer, HMW }\end{array}$ & $7.2 \pm 0.3^{d}$ & $0.56 \pm 0.01^{\mathrm{c}}$ & $3.87 \pm 0.08^{\mathrm{c}}$ & $56^{\mathrm{c}}$ & $44^{c}$ & 78 & 538 \\
\hline $\begin{array}{l}\text { PccGCS } \\
\text { Dimer }\end{array}$ & Dimer & N.D. & $0.67 \pm 0.02^{c}$ & $4.7 \pm 0.3^{c}$ & $56^{c}$ & $44^{c}$ & N.D. & N.D. \\
\hline PccGlobin & Dimer & $6.1 \pm 0.3$ & $0.66 \pm 0.01$ & $5.8 \pm 0.1$ & 79 & 21 & 108 & 944 \\
\hline $\begin{array}{l}\text { PccGlobin } \\
\text { (S82A) }\end{array}$ & Dimer & $7.9 \pm 0.5$ & $3.02 \pm 0.01$ & - & 100 & - & 382 & - \\
\hline $\begin{array}{l}\text { PccGlobin } \\
\text { (Y57F) }\end{array}$ & Dimer & - & - & - & - & - & - & - \\
\hline $\begin{array}{l}\text { PccGlobin } \\
\text { (F148R) }\end{array}$ & $\begin{array}{l}\text { Monomer, } \\
\text { dimer }\end{array}$ & $7.4 \pm 0.1$ & $1.22 \pm 0.01$ & $6.98 \pm 0.07$ & 86 & 14 & 165 & 943 \\
\hline
\end{tabular}

- Not observed

N.D., not determined

HMW, high molecular weight (octamer and larger)

${ }^{a}$ All errors for $\% k_{1}$ and $\% k_{2}$ are $\leq 5 \%$

${ }^{\mathrm{b}}$ Ref. [29]

${ }^{\mathrm{c}}$ Ref. [17]

${ }^{\mathrm{d}}$ Ref. [16] 
The effect of salt concentration on $P c c$ Globin $\mathrm{O}_{2}$ dissociation rates also was measured to determine if the higher salt concentrations, which can be accessed using stopped flow as compared to AGF (1 M vs. $300 \mathrm{mM}$, respectively), would weaken salt bridges putatively stabilizing the globin dimer interface. Despite the high salt concentration, no change in $\mathrm{O}_{2}$ dissociation rates or rate percentages was observed (Table 2). These data further support the AGF data that show that the PccGlobin dimer interface has high affinity and is a key determinant of dimer formation in full-length PccGCS.

Table 2. $P$ ccGlobin WT salt-dependent $\mathrm{O}_{2}$ dissociation rates.

\begin{tabular}{|l|c|l|c|c|}
\hline Salt concentration & $\boldsymbol{k}_{\mathbf{1}}\left(\mathbf{s}^{\mathbf{- 1}}\right)$ & \multicolumn{1}{|c|}{$\boldsymbol{k}_{\mathbf{2}}\left(\mathbf{s}^{\mathbf{- 1}}\right)$} & $\boldsymbol{\%} \boldsymbol{k}_{\mathbf{1}}$ & $\boldsymbol{\%} \boldsymbol{k}_{\mathbf{2}}$ \\
\hline $50 \mathrm{mM} \mathrm{NaCl}$ & $0.681 \pm 0.002$ & $5.48 \pm 0.08$ & $82.88 \pm 0.5$ & $17.12 \pm 0.5$ \\
\hline $300 \mathrm{mM} \mathrm{NaCl}$ & $0.598 \pm 0.001$ & $3.5 \pm 0.1$ & $79.39 \pm 0.8$ & $20.61 \pm 0.8$ \\
\hline $500 \mathrm{mM} \mathrm{NaCl}$ & $0.584 \pm 0.002$ & $4.64 \pm 0.08$ & $82.88 \pm 0.4$ & $17.12 \pm 0.4$ \\
\hline $750 \mathrm{mM} \mathrm{NaCl}$ & $0.543 \pm 0.002$ & $4.0 \pm 0.2$ & $82.27 \pm 1.2$ & $17.73 \pm 1.2$ \\
\hline $1000 \mathrm{mM} \mathrm{NaCl}$ & $0.563 \pm 0.001$ & $5.01 \pm 0.05$ & $80.67 \pm 0.5$ & $19.33 \pm 0.5$ \\
\hline
\end{tabular}

Measurement of $P c c$ Globin(F148R) $\mathrm{O}_{2}$ dissociation rates in varying salt concentrations found that $0 \mathrm{mM} \mathrm{NaCl}$ resulted in $k_{1}$ and $k_{2}$ percentages similar to WT PccGlobin, likely due to increased dimer formation, while inclusion of salt decreased the percentage of $k_{2}$ (Tables 1 and 3), potentially by stabilizing monomeric PccGlobin(F148R). These data highlight the importance of the globin dimer interface in controlling conformations of the heme pocket and $\mathrm{O}_{2}$ affinity within globin coupled sensors. An additional mutation (S155R) putatively located at the center of the dimer interface was generated to further disrupt PccGlobin dimerization However, this mutation resulted in unstable protein that readily aggregated, prohibiting analysis of the effects of dimerization on $\mathrm{O}_{2}$ binding kinetics. 
To further characterize the effects of globin dimerization on the heme pocket, $\mathrm{O}_{2}$ association rates were measured (Table 1). PccGlobin exhibited an association rate $\left(k_{\mathrm{on}}\right)$ almost identical to full-length PccGCS $\left(6.1 \mu \mathrm{M}^{-1} \mathrm{~s}^{-1}\right.$ and $7.2 \mu \mathrm{M}^{-1} \mathrm{~s}^{-1}{ }^{16}$, respectively), demonstrating that removing the middle and diguanylate cyclase domains, but maintaining the globin dimer interface, does not significantly affect $\mathrm{O}_{2}$ entry into the heme pocket. In contrast, BpeGlobin exhibited a markedly faster $\mathrm{O}_{2}$ association rate, as compared to full-length BpeGReg $\left(17.6 \mu \mathrm{M}^{-1} \mathrm{~s}^{-1}\right.$ vs. $7.0 \mu \mathrm{M}^{-1} \mathrm{~s}^{-1} 29$, respectively). The biphasic $\mathrm{O}_{2}$ dissociation rates yield calculated $\mathrm{O}_{2}$ affinities of $108 \mathrm{nM}$ and 944 $\mathrm{nM}$ for PccGlobin, as compared to $78 \mathrm{nM}$ and $538 \mathrm{nM}$ for full-length PccGCS. In contrast, BpeGlobin yields a single $67 \mathrm{nM} \mathrm{O}$ affinity, while $\mathrm{O}_{2}$ affinities of $117 \mathrm{nM}$ and $900 \mathrm{nM}$ were calculated for full-length BpeGReg. Dual $\mathrm{O}_{2}$ affinities in both full-length proteins may allow the organisms to sense a wide range of $\mathrm{O}_{2}$ concentrations, allowing the bacteria to modulate their responses to adapt to moderate and then low $\mathrm{O}_{2}$ levels.

Table 3. $P c c$ Globin(F148R) salt-dependent $\mathrm{O}_{2}$ dissociation rates.

\begin{tabular}{|l|c|c|c|c|}
\hline Salt Concentration & $\mathbf{k}_{\mathbf{1}}\left(\mathbf{s}^{-\mathbf{1}}\right)$ & $\mathbf{k}_{\mathbf{2}}\left(\mathbf{s}^{\mathbf{- 1}}\right)$ & $\mathbf{\%} \mathbf{k}_{\mathbf{1}}$ & $\boldsymbol{\%} \mathbf{k}_{\mathbf{2}}$ \\
\hline $0 \mathrm{mM} \mathrm{NaCl}$ & $1.06 \pm 0.002$ & $6.38 \pm 0.04$ & $80.5 \pm 2.4$ & $19.5 \pm 2.4$ \\
\hline $50 \mathrm{mM} \mathrm{NaCl}$ & $1.224 \pm 0.002$ & $6.98 \pm 0.07$ & $85.9 \pm 0.5$ & $14.1 \pm 0.5$ \\
\hline $150 \mathrm{mM} \mathrm{NaCl}$ & $1.203 \pm 0.002$ & $7.43 \pm 0.09$ & $86.2 \pm 1.1$ & $13.8 \pm 1.4$ \\
\hline $300 \mathrm{mM} \mathrm{NaCl}$ & $1.226 \pm 0.003$ & $6.97 \pm 0.08$ & $85.5 \pm 1.9$ & $14.5 \pm 1.9$ \\
\hline
\end{tabular}

As truncating PccGCS did not result in a change in $\mathrm{O}_{2}$ association rate, it is unlikely that the change in rate for BpeGlobin is due to the loss of the middle and cyclase domains. Instead, loss of the globin dimerization interface may be responsible for the 2.5-fold increase in the rate of $\mathrm{O}_{2}$ binding. Given that crystal structures of homologous sensor globins solved as dimers found that 
entry to the heme pocket was away from the globin-globin interface ${ }^{28,30}$ (Fig. 1), the changes in $k_{\text {on }}$ are unlikely to be due to $\mathrm{O}_{2}$ access being blocked by the dimer interface. Instead, both the $\mathrm{O}_{2}$ association and dissociation rate data for BpeGlobin suggest that loss of the globin dimer interface results in significant conformational changes to the heme pocket, increasing accessibility and altering conformations/dynamics within the pocket.

\subsection{Involvement of Distal Pocket Residues in Biphasic $\mathrm{O}_{2}$ Dissociation Rates}

Based on crystal structures and sequence alignments, PccGlobin and BpeGlobin are proposed to have two amino acids (Y57/S82 and Y43/S68, respectively) within the distal pocket that serve as hydrogen bond donors and stabilize $\mathrm{O}_{2}$ binding (Fig. 1A, S2) ${ }^{17-18,28,30}$. Within HemAT-Bs, both the distal pocket tyrosine and threonine contribute to $\mathrm{O}_{2}$ stabilization, while EcDosC only has a distal tyrosine to provide hydrogen bonding ${ }^{18,30-32}$. Therefore, mutations of both distal pocket residues within PccGlobin and BpeGlobin were analyzed to determine which residue is involved in the fast $\mathrm{O}_{2}$ dissociation rate $\left(k_{2}\right)$.

Given the rapid autoxidation, $\mathrm{O}_{2}$ association and dissociation rates for PccGlobin(Y57F) and BpeGlobin(Y43F) could not be obtained. In contrast, both serine mutants, PccGlobin(S82A) and BpeGlobin(S68A), were able to bind $\mathrm{O}_{2}$; however, even though PccGlobin(S82A) forms a dimer (Fig. 4D), the $\mathrm{O}_{2}$ dissociation rate simplified to a single exponential (Table 1), suggesting that the distal pocket serine is involved in interactions with bound $\mathrm{O}_{2}$ and/or distal pocket residues that lead to biphasic kinetics and the fast $\mathrm{O}_{2}$ dissociation rate $\left(k_{2}\right)$. Mutation of PccGlobin distal pocket serine also leads to an increase in $k_{1}$, which is not surprising, given that a secondary 


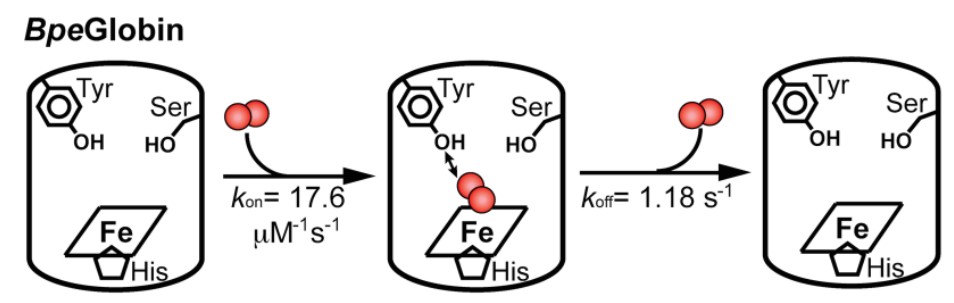

\section{PccGlobin}

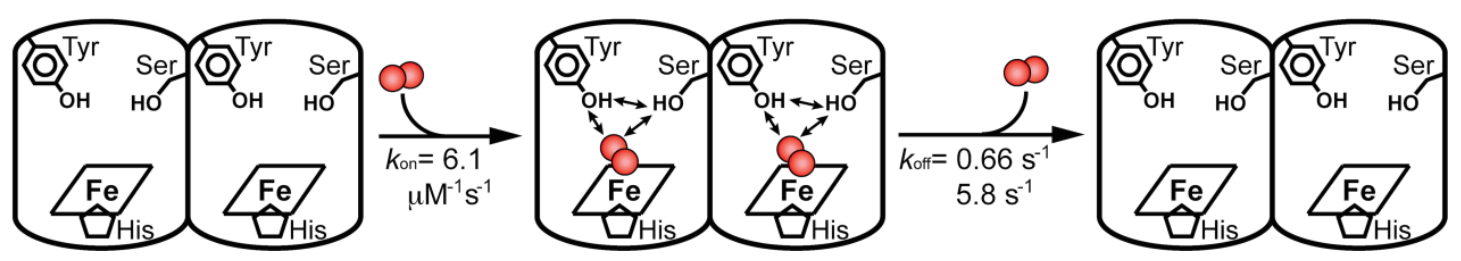

Figure 5. Overview of the effects of globin dimerization on $\mathrm{O}_{2}$ binding kinetics. Monomeric BpeGlobin (top) exhibits a single $\mathrm{O}_{2}$ dissociation rate, with the distal pocket tyrosine serving as the primary hydrogen bond donor. In contrast, dimeric PccGlobin (bottom) exhibits two $\mathrm{O}_{2}$ dissociation rates, the faster rate requiring the presence of the distal pocket serine. Dimerization causes a conformational change in the distal pocket, positioning the serine such that it affects $\mathrm{O}_{2}$ dissociation through interactions with bound $\mathrm{O}_{2}$ and/or the distal pocket tyrosine.

hydrogen-bonding residue has been removed, and is similar to the increase observed for BpeGlobin(S68A). The $\mathrm{O}_{2}$ association rates for the serine mutants of PccGlobin and BpeGlobin did not change relative to the WT proteins ${ }^{16,29}$ (Table 1), further confirming that the mutations did not significantly affect the heme pocket. These data support the hypothesis that globin domain dimerization leads to conformation(s) of the distal pocket that adjust the position of the hydrogen bonding residues, leading to increased interaction with the distal pocket serine and resulting in biphasic $\mathrm{O}_{2}$ dissociation (Fig. 5). 


\section{Conclusions}

In conclusion, globin domains from PccGCS and BpeGReg exist in different oligomeric states, despite the relatively high amino acid sequence similarity at the putative dimer interface. These differences in oligomerization result in altered properties of the heme domains. Globin domain dimerization within globin coupled sensors leads to biphasic $\mathrm{O}_{2}$ dissociation, likely through changes in the conformation of the heme pocket. Of the two distal pocket hydrogen bond donors, tyrosine provides direct hydrogen bonding, while a serine residue serves as a secondary hydrogen bond donor. In addition, PccGlobin distal pocket serine makes key interactions involved in the second, fast $\mathrm{O}_{2}$ dissociation rate observed in dimeric sensor globins, suggesting that dimerization changes the accessible heme pocket conformations and position of the serine. These data provide insight into the oligomerization determinants of the full-length GCS proteins and information about the oligomerization-dependent changes to the heme pocket GCS globin domains.

\section{Abbreviations:}

AGF - Analytical gel filtration

BpeGlobin - Globin domain from BpeGReg

BpeGReg - Globin Coupled Sensor from B. pertussis

C-di-GMP - cyclic diguanosine monophosphate

GCS - Globin Coupled Sensor

GTP - Guanosine triphosphate

HPLC - High Pressure Liquid Chromatography

$\mathrm{O}_{2}$ - Oxygen

PccGCS - Globin Coupled Sensor from P. carotovorum 
PccGlobin - Globin domain from PccGCS

WT - Wild type

Acknowledgments. This work was supported by a US National Science Foundation CAREER

Grant CHE 1352040 (E.E.W.), a University Research Committee Grant from Emory University (E.E.W), and a Laney Graduate School Fellowship (S.R.). The authors are grateful to Professor R.B. Dyer (Emory University) for assistance with $\mathrm{O}_{2}$ association rate measurements and members of the Weinert lab for helpful discussions.

\section{References}

[1] E. Karatan, P. Watnick, Microbiol. Mol. Biol. Rev. 73 (2009), 2, 310-47.

[2] D. Srivastava, C.M. Waters, J. Bacteriol. 194 (2012), 17, 4485-93.

[3] H. Cypionka, Annu. Rev. Microbiol. 54 (2000), 827-48.

[4] H. Sass, M. Berchtold, J. Branke, H. Konig, H. Cypionka, H.D. Babenzien, Syst. Appl. Microbiol. 21 (1998), 2, 212-219.

[5] C. Wu, Y.Y. Cheng, H. Yin, X.N. Song, W.W. Li, X.X. Zhou, L.P. Zhao, L.J. Tian, J.C. Han, H.Q. Yu, Sci. Rep. 3 (2013), 1945.

[6] C.E. Bauer, S. Elsen, T.H. Bird, Annu. Rev. Microbiol. 53 (1999), 495-523.

[7] M.A. Gilles-Gonzalez, IUBMB Life 51 (2001), 3, 165-73.

[8] J. Green, J.C. Crack, A.J. Thomson, N.E. LeBrun, Curr. Opin. Microbiol. 12 (2009), 2, $145-51$.

[9] T. Shimizu, D. Huang, F. Yan, M. Stranava, M. Bartosova, V. Fojtikova, M. Martinkova, Chem. Rev. 115 (2015), 13, 6491-533. 
[10] S.N. Vinogradov, M. Tinajero-Trejo, R.K. Poole, D. Hoogewijs, Biochim. Biophys. Acta 1834 (2013), 9, 1789-800.

[11] M. Martinkova, K. Kitanishi, T. Shimizu, J. Biol. Chem. 288 (2013), 39, 27702-11.

[12] S. Hou, C. Belisle, S. Lam, M. Piatibratov, V. Sivozhelezov, H. Takami, M. Alam, Extremophiles 5 (2001), 5, 351-4.

[13] S. Hou, T. Freitas, R.W. Larsen, M. Piatibratov, V. Sivozhelezov, A. Yamamoto, E.A. Meleshkevitch, M. Zimmer, G.W. Ordal, M. Alam, Proc. Natl. Acad. Sci. U.S.A. 98 (2001), 16, 9353-8.

[14] S. Hou, R.W. Larsen, D. Boudko, C.W. Riley, E. Karatan, M. Zimmer, G.W. Ordal, M. Alam, Nature 403 (2000), 6769, 540-4.

[15] K. Kitanishi, K. Kobayashi, T. Uchida, K. Ishimori, J. Igarashi, T. Shimizu, J. Biol. Chem. 286 (2011), 41, 35522-34.

[16] X. Jia, J.B. Wang, S. Rivera, D. Duong, E.E. Weinert, Nature Commun. 7 (2016), 12381.

[17] J.L. Burns, D.D. Deer, E.E. Weinert, Mol. Biosyst. 10 (2014), 11, 2823-6.

[18] K. Kitanishi, K. Kobayashi, Y. Kawamura, I. Ishigami, T. Ogura, K. Nakajima, J. Igarashi, A. Tanaka, T. Shimizu, Biochemistry 49 (2010), 49, 10381-93.

[19] H. Sawai, S. Yoshioka, T. Uchida, M. Hyodo, Y. Hayakawa, K. Ishimori, S. Aono, Biochim. Biophys. Acta 1804 (2010), 1, 166-72.

[20] J.R. Tuckerman, G. Gonzalez, E.H. Sousa, X. Wan, J.A. Saito, M. Alam, M.A. GillesGonzalez, Biochemistry 48 (2009), 41, 9764-74.

[21] R. Hengge, Nature Rev. Microbiol. 7 (2009), 4, 263-73.

[22] U. Romling, M.Y. Galperin, M. Gomelsky, Microbiol. Mol. Biol. Rev. 77 (2013), 1, 152. 
[23] C. Chan, R. Paul, D. Samoray, N.C. Amiot, B. Giese, U. Jenal, T. Schirmer, J. Deisenhofer, Proc. Natl. Acad. Sci. U.S.A. 101 (2004), 49, 17084-17089.

[24] A. Deepthi, C.W. Liew, Z.-X. Liang, K. Swaminathan, J. Lescar, PloS One 9 (2014), 10, e110912.

[25] N. De, M.V.A.S. Navarro, R.V. Raghavan, H. Sondermann, J. Mol. Biol. 393 (2009), 3, 619-633.

[26] R. Paul, S. Abel, P. Wassmann, A. Beck, H. Heerklotz, U. Jenal, J. Biol. Chem. 282 (2007), 40, 29170-29177.

[27] P. Wassmann, C. Chan, R. Paul, A. Beck, H. Heerklotz, U. Jenal, T. Schirmer, Structure 15 (2007), 8, 915-927.

[28] M. Tarnawski, T.R. Barends, I. Schlichting, Acta Crystallogr. D Biol. Crystallogr. 71 (2015), Pt 11, 2158-77.

[29] X. Wan, J.R. Tuckerman, J.A. Saito, T.A. Freitas, J.S. Newhouse, J.R. Denery, M.Y. Galperin, G. Gonzalez, M.A. Gilles-Gonzalez, M. Alam, J. Mol. Biol. 388 (2009), 2, 262-70.

[30] W. Zhang, G.N. Phillips, Structure 11 (2003), 9, 1097-1110.

[31] T. Ohta, H. Yoshimura, S. Yoshioka, S. Aono, T. Kitagawa, J. Am. Chem. Soc. 126 (2004), 46, 15000-1.

[32] W. Zhang, J.S. Olson, G.N. Phillips, Jr., Biophys. J. 88 (2005), 4, 2801-14.

[33] C. Camacho, G. Coulouris, V. Avagyan, N. Ma, J. Papadopoulos, K. Bealer, T.L. Madden, BMC Bioinformatics 10 (2009), 421.

[34] E.E. Weinert, C.M. Phillips-Piro, R. Tran, R.A. Mathies, M.A. Marletta, Biochemistry 50 (2011), 32, 6832-40. 
[35] E.E. Weinert, L. Plate, C.A. Whited, C. Olea, Jr., M.A. Marletta, Angew. Chem. Int. Ed. Engl. 49 (2010), 4, 720-3.

[36] B.L. Greene, C.H. Wu, P.M. McTernan, M.W. Adams, R.B. Dyer, J. Am. Chem. Soc. 137 (2015), 13, 4558-66.

[37] L.A. Kelley, S. Mezulis, C.M. Yates, M.N. Wass, M.J. Sternberg, Nature Protoc. 10 (2015), 6, 845-58. 

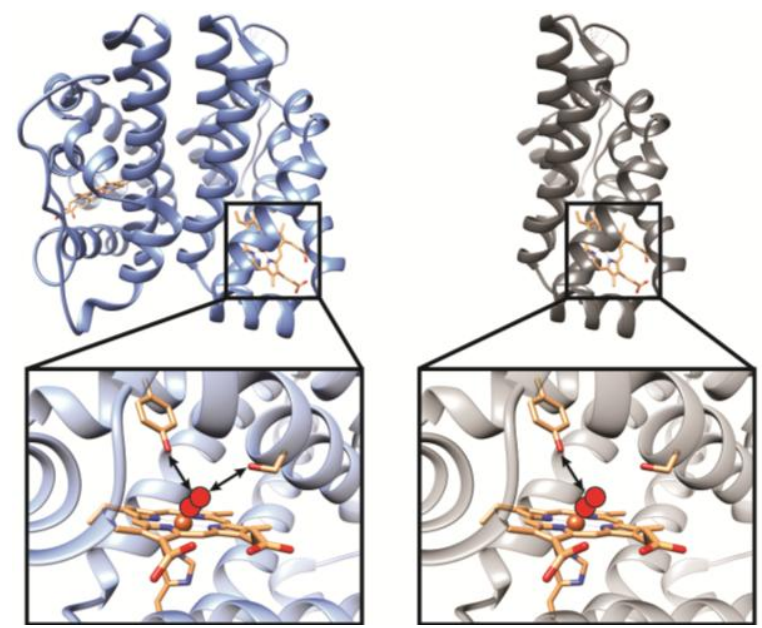

Graphical Abstract and Synopsis

Bacteria use globin coupled sensors (GCS) to sense and response to changing oxygen levels. Globin domains with high amino acid similarity display differential oligomerization and oxygen binding kinetics. Dimerization of the globin domain within GCS proteins results in changes to the heme pocket, altering the conformation and oxygen dissociation kinetics. 